Research Article

\title{
Anti-genotoxic and anti-inflammatory effects of ethanol extract of stoechospermum marginatum (c. agardh) kutzing in rats
}

\begin{abstract}
Stoechospermum marginatum (C. Agardh) Kutzing is belongs to brown algae distributed from Indian Ocean to Australian ocean. It was obtained from the Gulf of Mannar near Mandapam coast $\left(9^{\circ} 16^{\prime} \mathrm{N}, 79^{\circ} 12^{\prime} \mathrm{E}\right)$ Tamilnadu. There was no novel scientific record as like in our present study so far. Therefore, in the present imperative pragmatic study revealed about the anti-genotoxic and anti-inflammatory activities of ethanol extract of $S$. marginatum in rats. For the anti genotoxicity, the reference genotoxin 4-NQO was adapted to induce genotoxic damage in terms of micronuclei in rat bone marrow cells via micronuclei assay scored using light microscopy. Acute inflammatory study was carried out by inducing paw edema through carrageenan injection in rat right hind paw. Subsequently, the chronic inflammation experiments were also carried out by stimulating granulomatous tissue mass around the cotton pellets implanted subcutaneously in the dorsal region of the rats. The pragmatic results showed that 4-NQO enhanced the MnPCEs frequency about $67 \pm 5.79$ which was 4.5 fold higher than that of the control value, 15.0 2.23 MnPCEs/2500PCEs. Ethanol extract of $S$. marginatum alone treated group did not shown any changes in the frequency of MnPCEs $(16.0 \pm 1.58, \mathrm{P}>0.05)$. However, pretreatment with ethanol extract of $S$. marginatum plus 4-NQO treated groups were observed to be significantly reduced the frequency of MnPCEs ranging from about 12 to $73 \%$. Similarly, paw volume in the control group was observed to be enhanced about $0.42 \pm 0.08$ to $0.52 \pm 0.08$ at time interval of $1-8 \mathrm{hrs}$. Standard drug of diclofenac and ethanol extract of $S$. marginatum were also observed to be decrease the paw volume significantly based on the dose with time interval. Therefore, this imperative study concluded that the anti genotoxic and anti-inflammatory activities of ethanol extract of $S$. marginatum were significantly exhibited dose dependent effects as equally as standard drug of diclofenac.
\end{abstract}

Keywords: stoechospermum marginatum, ethanol extract, 4-NQO, anti-genotoxicity, anti-inflammatory
Volume 8 Issue 4 - 2019

\author{
Arumugam $\mathrm{P},{ }^{\prime}$ Arunkumar $\mathrm{K},{ }^{2}$ Murugan $\mathrm{M},{ }^{3}$ \\ Murugan $\mathrm{K}^{\prime}$ \\ 'Department of Zoology, Bharathiar University, India \\ 2Department of Plant Science, Bharathiar University, India \\ ${ }^{3}$ Department of Microbial Technology, Bharathiar University, \\ India
}

Correspondence: Ponnan Arumugam, Department of Zoology, School of Life Science, Bharathiar University,

Coimbatore - 64l 046, Tamil Nadu, India, Tel +9l 8939433020 , Email ponnanarumugam@gmail.com

Received: June 25, 2019 | Published: August 21, 2019
Abbreviations: MnPCEs, micro nucleated polychromatic erythrocytes; Ac-4-HAQO, active form of 4-Hydroxyl-aminoquinoline-1-oxide; NCEs, normochromatic erythrocytes; CPCSEA, control and supervision of experiments on animals; $\mathrm{AF}$, aqueous fractions; DMF, dichloromethane fractions; ECF, ethyl acetate fractions

\section{Introduction}

Marine organisms are having rich source biologically active metabolites and there are many reports of macro algae-derived compounds that posses a broad range of biological functions, such as antibiotic, antiviral, antioxidant, antifouling, anti-inflammatory, cytotoxic, and anti-mitotic activities. ${ }^{1,2}$ Some of the marco algaederived bioactive compounds are amino acids, terpenoids, phlorotannins, steroids, alkaloids, polyketides, glycerols, cyclic peptide, polysaccharides, phenolic compounds, fatty acids, and acrylic acid. ${ }^{3,4}$ These bioactive potential of seaweeds are mainly used as a human food sources in Asian countries. Particularly, brown seaweed (Phaephyceae) is most complex group of algae and they are broadly distributed from tropical to polar zones of ocean in the world. ${ }^{5}$ Stoechospermum marginatum (C. Agardh) Kutzing belongs to brown algae and distributed from Indian Ocean to Australian ocean. ${ }^{6}$ It quiet effortlessly collected from the Gulf of Mannar near Mandapam coast $\left(9^{\circ} 16^{\prime} \mathrm{N}, 79^{\circ} 12^{\prime} \mathrm{E}\right)$ of Tamilnadu, India. Currently, this brown seaweed utilized for various aspects like used as a food in salads, fresh meal for breeding animals, manure for cultivation of vegetable and raw material for production of alginic acid and mannitol. ${ }^{7}$ Different essential oils along with methanolic and dichloromethane (1:1) extract of $S$. marginatum possessed antioxidant and antibacterial potential were also documented. ${ }^{3}$ Recent report revealed that the secondary metabolites present in $S$. marginatum were noticed to be boosted the activities of peroxidase, phenyl alanine lyase, catalyse, and poly phenol oxidase. ${ }^{8}$ Spatane derivatives from $S$. marginatum were well established regarding their biological activity. ${ }^{4}$ However, bioactive composition was differed depending on the geographical distribution, habitats including environmental conditions: water, temperature, salinity, light, and nutrients. ${ }^{9}$ In the present novel study was seems to be lacking based on the review of literature and therefore in the present pragmatic study intended to evaluate the anti-genotoxic and anti-inflammatory effects of Stoechospermum marginatum ethanol extract in rat model.

\section{Materials and methods}

\section{Chemicals and solvents}

Following chemicals such as indomethacin, giemsa stain, maygrunwald stain and well-know genotoxin, 4-nitroquinoline-1-oixde (4-NQO) were procured from Sigma-Aldrich, USA. Other liquid solution like phosphate buffered saline and DMSO purchased through Hi-media, Mumbai, India. All the solvents used in the present study were of analytical grade. 


\section{Collection and extraction of Stoechospermum marginatum}

The brown seaweed of $S$. marginatum (J. Agardh) Kuetz was composed in place where the intertidal zone of Mandapam, Gulf of Mannar, and South-east Coast of India. The collected fresh seaweed of $1 \mathrm{~kg}$ was washed thoroughly with tap water and subsequently with distilled water to remove superfluous materials such as salt, debris and necrotic parts. Then, the washed seaweed shade dried and powdered through mixer grinder. Solvent extraction and fractionations has been carried out based on our previous report. ${ }^{10}$ All the solvent extraction and fractions were concentrated using rotary evaporator where as aqueous fraction by lyophilizer. The yield of ethanol extract was obtained about $20 \mathrm{~g}(8 \%)$ whereas hexane (HF) dichloromethane (DMF), ethyl acetate (EAF) and aqueous fractions (AF) were about $25 \%, 35 \%, 30 \%$ and $10 \%$ respectively. However, in the in-vivo bioactivity of ethanol extract of S. marginatum was examined in this article instated of other fractions based on the previous investigation. ${ }^{2}$

\section{Animal experimental design}

Either sex of Wistar albino rat $(150-180 \mathrm{~g})$ was commercially purchased and accommodated in the laboratory condition $(12 \mathrm{~h}$ light $/ 12 \mathrm{~h}$ dark; $22 \pm 2^{\circ} \mathrm{C}$ ) for the period of one week. All the rats were freely adapted to consume tap water and rodent pellet diet. Hence, the experimental designs and execution were followed by the guidelines of the Committee for the Purpose of Control and Supervision of Experiments on Animals (CPCSEA), India

\section{Rat model for anti-genotoxicity and anti-inflammatory}

Micronucleus assay for anti-genotoxicity: Either sex of Wistar albino rats was alienated into six groups with each five rats. The concentrated ethanol extract of $S$. marginatum was suspended in $15 \%$ DMSO and fed by orally to the experimental rats up to five consecutive days. Fist group considered as a control fed with DMSO by orally. Second group also received high dose of ethanol extract of $S$. marginatum, $640 \mathrm{mg} / \mathrm{kgbw}$. The well-known genotoxin, 4-NQO (7.5mg/kgbw) was considered as group third fed by intra peritoneally. Similarly, other groups received respective test doses 160,320 , and $640 \mathrm{mg} / \mathrm{kgbw}$ of ethanol extract of $S$. marginatum up to five consecutive days plus $6^{\text {th }}$ day 4-NQO (7.5mg/kgbw) by IP. After 24 hours of 4-NQO treatment, all the experimental animals were sacrificed by cervical dislocation. The kneecap was gently cut and the marrow cells of the femur bones was flushed into $2 \mathrm{~mL}$ fetal bovine serum for the micronucleus assay based on the protocol reported in our previous publication. ${ }^{11}$ There are 2,500 polychromatic erythrocytes (with or without micronuclei) and a corresponding number of normal chromatic erythrocytes (NCEs) were scored in both experimental and control animal through light microscope

\section{Paw edema by carrageenan and granuloma by cotton pellet}

The experimental design consisted five groups and each group having five Wistar albino rats. All the groups such as control (15\% DMSO), three test doses of ethanol extract of $S$. marginatum (160, $320, \& 640 \mathrm{mg} / \mathrm{kg})$ and positive control of diclofenac $(25 \mathrm{mg} / \mathrm{kg}$ in DMSO) were fed by orally. Subsequently, freshly prepared $0.1 \mathrm{~mL}$ of $1 \%$ carrageenan solution in normal saline was injected in the right hind paw of all the experimental rats exactly after 1hour of the test doses administration. Before and after injection of carrageenan, the hind paw swelling was measured (as $\mathrm{cm}$ ) for the time interval of 1,2 , 4 and 8hours through Plethysmograph. Thus, the measured hind paw volume of acute inflammation was expressed as \% inhibition of paw volume by both standard and ethanol extract of $S$. marginatum over the control group. ${ }^{12}$

Similarly, the experimental design for granuloma induced by cotton pellet also consist five groups of five rats of either sex was used. Group one received 15\% DMSO as native control and second group received anti-inflammatory drugs, diclofenac as a positive control. Another three groups fed different of doses of ethanol extract of $S$. marginatum $(160,320, \& 640 \mathrm{mg} / \mathrm{kg})$ by orally every day up to one week to all the groups. Later, all the experimental rats on eighth day were anesthetized with slight ether. Subsequently implanted four sterile cotton pellets $(50 \mathrm{mg})$ in to the subcutaneous dorsal region of rats in which two in the axilla region and another two in the groin region. End of the experiments ( $16^{\text {th }}$ day), all the experimental rats were sacrificed under the slight anesthetic ether. Implanted cotton pellets were removed without interrupting the granuloma tissues deposit around the cotton pellets. Accordingly, the fresh and dry weight of the cotton pellets were immediately regarded with wet pellet cotton and dried pellet cotton underwent $60^{\circ} \mathrm{C}$ for $48 \mathrm{~h} .{ }^{12}$ The degree of observed cotton pellet weights values for the test compounds and standard drug were made the statistics by compared with respective negative and positive controls.

\section{Statistical analysis}

In the pragmatic study results were all expressed as mean \pm standard deviation for five rats of each group. All the experimental values were performed statistical analyses by one-way ANOVA using SPSS Software (Version 16.0). The differences among the groups were brought out through Student-Neuman-Keuls test. $p \leq 0.05$ values were considered to be significant.

\section{Results and discussion}

The core value of this research article is certainly linked with bioactive potential in terms of brown seaweed such as $S$. marginatum. First part of this article revealed about the anti genotoxic effects of ethanol extract of S.marginatum against 4-NQO induced micro nucleated polychromatic erythrocytes (MnPCEs) in rat bone marrow cells were thoroughly evaluated and their results depicted in Figure 1. The ethanol extract of $S$. marginatum was used in this pragmatic study in terms of high antioxidant potential of the ethanol extract than that of its solvent fractions published in our pervious articles. ${ }^{2}$ The well-known genotoxin, 4-NQO was employed to induce genotoxicity in rat bone marrow cells. However, it exerts genotoxicity through forming serious of metabolites in which the core more active form of 4-hydroxyaminoquinoline 1-oxide (Ac-4-HAQO) was exert the toxicity by forming DNA adduct at the N2, C8 and of N6 position. ${ }^{13,14}$ As results, the helical structure of the DNA undergo breakdown and become in terms of micronuclei/chromosomal breakage/fragments. ${ }^{15}$ During the cell cycle progression, small fragments are not able included in the anaphase stage when the centric elements move towards the spindle poles. However, the undamaged chromosomes and centric fragments are included after telophase in the daughter cells. Small fragments of lagged chromosome later transform into one or several secondary nuclei called micronuclei, which are much smaller by about $1 / 5-1 / 20^{\text {th }}$ the size of the main nucleus. 


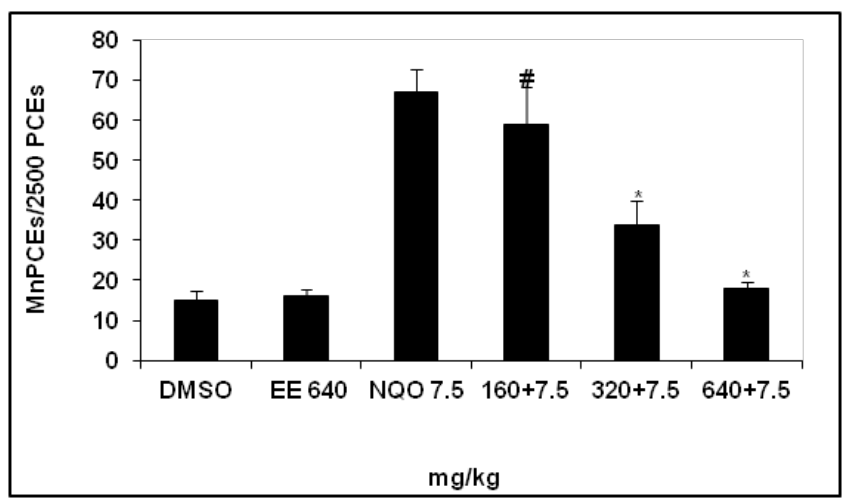

Figure I Anti genotoxic effect of ethanol extract of Stoechospermum marginatum on 4-NQO induced micronuclei in rat bone marrow cells (DMSO: control; EE: ethanol extract; NQO: 4-nitroquinoline-I- oxide: $*_{p} \leq 0.05$; Comparisons were made between control and treated groups)

A few hours after final stage of mitosis, mature erythroblasts eject its main nucleus however the cytoplasm of the young erythrocyte having micronuclei that can be easily visualized as a small spherical bodies. While staining, mature erythrocytes look as yellowish brown so called as normochromatic erythrocytes (NCEs) and young erythrocytes seem to be bluish so called as polychromatic erythrocytes (PCEs). ${ }^{11}$ In the pragmatic study results about the anti-genotoxicity of ethanol extract of $S$. marginatum were revealed the estimation of the frequency of micro nucleated PCEs (MnPCEs) in the bone marrow of the treated animals in comparison with the untreated animals. The results of well-known genotoxin, 4-NQO enhanced the frequency of MnPCEs $(67 \pm 5.79)$ about 4.5 times higher than the control value, 15.0 $\pm 2.23 \mathrm{MnPCEs} / 2500 \mathrm{PCEs}$. There was no changes in the frequency of MnPCEs $(16.0 \pm 1.58, P>0.05)$ in the ethanol extract of $S$. marginatum alone treated group.

Whereas, pretreatment with ethanol extract of $S$. marginatum followed by 4-NQO treated groups were found to be significantly reduced the 4-NQO enhanced MnPCEs frequency about 12 to $73 \%$. Hence, the highest reduction against 4-NQO enhanced MnPCEs was observed at the dose of $640 \mathrm{mg} / \mathrm{kgbw}$ (about $73 \%$ ) and lowest at the dose of $160 \mathrm{mg} / \mathrm{kgbw}$ (about $12 \%$ ). Among them, the maximum dose showed 3.3 fold better reductions against 4-NQO enhanced MnPCEs than that of their lower dose. The second highest dose $320 \mathrm{mg} / \mathrm{kgbw}$ of ethanol extract of $S$. marginatum was also extensively reduced the MnPCEs frequency (about 49\%) against 4-NQO enhanced MnPCEs that was regarded to be 1.7 fold higher MnPCEs reduction over the lower dose, $160 \mathrm{mg} / \mathrm{kgbw}$. The entire pragmatic investigation about the anti-genotoxicity of ethanol extract of $S$. marginatum was proved that it significantly $(P<0.05)$ protected the $4-\mathrm{NQO}$ induced MnPCE frequency in a dose dependent manner.

In general, inflammations taking place due to when the cell/ tissue undergoes damage either by stress or physical injury in terms of that the inflammatory mediators such as histamine, bradykinin, serotonine and prostaglandins are released. As a results, inflammatory mediators boost the production of inflammation and nociceptors by the induction of pain. ${ }^{16}$ It is also localized further in tissues with high content of water and plasma due to the arachidonic acid metabolism in terms of cyclo-oxygenase and lipo-oxygenase enzyme pathways. ${ }^{17}$
Inflammation takes place within an hour as soon as injection of carrageenan and there by the release of histamine and serotonin. However, secondary inflammation also takes place after one hour of injection of carrageenan and there by the release of bradykinin, protease and prostaglandins. ${ }^{18}$

Prolonged inflammation can also be one the cause of genetic diseases including cancer. Therefore, this pragmatic investigation on acute anti-inflammatory activity of ethanol extract of $S$. marginatum was carried out against carrageenan induced paw volume in Wistar albino rats. This experiments consisted of five groups such as different dose $(160,320, \& 640 \mathrm{mg} / \mathrm{kg})$ of ethanol extract of $S$. marginatum, positive control of diclofenac $(25 \mathrm{mg} / \mathrm{kg}$ in DMSO) and negative control (DMSO) and each group having five rats. Carrageenan can be used for the stimulation of inflammation in terms of swelling of right hind paw. Carrageenan enhanced paw volume in rats were measured using plethysmograph at different time interval of 1,2, 4 and 8hours (Table 1). The results of negative control group were observed to be enhanced right hind paw volume about $0.42 \pm 0.08$ to $0.52 \pm 0.08$ at time interval of 1-8hrs. However, standard drug of diclofenac and ethanol extract of $S$. marginatum were found to be significanlty decreased the paw volume based on the dose with time interval enhanced by the carrageenan injection.

The decreased rate of the paw volume by diclofenac was about $27-62 \%$ with respect to the negative control group. In addition, ethanol extract of $S$. marginatum was also effectively decreasing the carrageenan induced paw volume. Among the dose tested, the highest dose $640 \mathrm{mg} / \mathrm{kg}$ bw was exhibited the maximum decrease rat of the paw volume about $23-57 \%$ at the time intervals of $1-8 \mathrm{hrs}$ followed by the dose of $320 \mathrm{mg} / \mathrm{kgbw}$ showed about $15-33 \%$ and the least was about $12-24 \%$ at the dose of $160 \mathrm{mg} / \mathrm{kg}$ bw of ethanol extract of $S$. marginatum. Thus, the ethanol extract of $S$. marginatum was significantly decrease the paw volume based on the degree of dose tested and their decreased grade as equal as standard of diclofenac.

The pragmatic study results about the chronic anti-inflammatory activity of ethanol extract of $S$. marginatum and standard antiinflammation drug of diclofenac were depicted in Table 2. The major pragmatic study results were regarded based on the cotton pellets weight surrounded by the mass of granulomatous tissue formed during the cotton pellets implanted subcutaneously in the dorsal region of the rats. Hence, the pragmatic results explored in terms of wet \& dry weight of cotton pellets of test substances such as ethanol extract of $S$. marginatum and positive control of diclofenac $(25 \mathrm{mg} / \mathrm{kg}$ in DMSO) over the negative control (DMSO). The percentage inhibition of granulomatous tissue mass formation by both anti-inflammatory drugs of diclofenac and ethanol extract of S. marginatum were found to be equally effective. Diclofenac inhibiting granulomatous tissue mass formation around the implanted cotton pellets was observed to be $66 \%$ over the negative control, $945 \pm 24.50 \mathrm{mg}$. Apart, ethanol extract of $S$. marginatum was also significantly (58\%) inhibiting granulomatous tissue formation around the implanted cotton pellets over the negative control. Among the tree tested doses, the highest dose alone showed effective chronic anti-inflammatory activity. Another two test doses were showed the percentage inhibition of granulomatous tissue formation in the range of $13-36 \%$ over the negative control. Though, chronic anti-inflammatory activity of ethanol extract of $S$. marginatum was also found to be dose dependent. 
Table I Anti-inflammatory potential of ethanol extract of Stoechospermum marginatum on carrageenan induced paw edema in rat

\begin{tabular}{|c|c|c|c|c|c|}
\hline \multirow{2}{*}{$\begin{array}{l}\text { Treatment } \\
\text { groups }\end{array}$} & \multirow[t]{2}{*}{ Oral dose (mg/kgbw) } & \multicolumn{4}{|c|}{ Paw volume at different time intervals $(h)$} \\
\hline & & I & 2 & 4 & 8 \\
\hline Control & DMSO & $0.52 \pm 0.08$ & $0.48 \pm 0.08$ & $0.46 \pm 0.06$ & $0.42 \pm 0.08$ \\
\hline \multirow[t]{2}{*}{$\mathrm{DCL}$} & 25 & $0.38 \pm 0.08 *$ & $0.30 \pm 0.10 *$ & $0.26 \pm 0.09 *$ & $0.16 \pm 0.06^{*}$ \\
\hline & & $27 \%$ & $38 \%$ & $44 \%$ & $62 \%$ \\
\hline \multirow{6}{*}{$\begin{array}{l}\text { Ethanol extract of } S \text {. } \\
\text { marginatum }\end{array}$} & 160 & $0.46 \pm 0.11 *$ & $0.42 \pm 0.08 *$ & $0.38 \pm 0.08^{*}$ & $0.32 \pm 0.08^{*}$ \\
\hline & & $12 \%$ & $13 \%$ & $17 \%$ & $24 \%$ \\
\hline & 320 & $0.44 \pm 0.09 *$ & $0.38 \pm 0.13^{*}$ & $0.3 \pm 0.05^{*}$ & $0.28 \pm 0.08 *$ \\
\hline & & $15 \%$ & $21 \%$ & $26 \%$ & $33 \%$ \\
\hline & 640 & $0.40 \pm 0.12 *$ & $0.36 \pm 0.05^{*}$ & $0.30 \pm 0.07 *$ & $0.18 \pm 0.08 *$ \\
\hline & & $23 \%$ & $25 \%$ & $35 \%$ & $57 \%$ \\
\hline
\end{tabular}

Values expressed in centimeters (mean \pm standard deviation). Percentages indicated to the change in paw volume with control group. Comparisons were made between control and drug treated groups. *p $\leq 0.05$ (DCL: Diclofenac; *significant).

Table 2 Effect of ethanol extract of Stoechospermum marginatum and diclofenac on cotton pellet induced chronic inflammation in rat

\begin{tabular}{|c|c|c|c|c|}
\hline \multirow{2}{*}{ Treatment groups (mg/kgbw) } & \multicolumn{2}{|l|}{ Cotton pellet } & \multirow{2}{*}{ Weight Difference (mg) } & \multirow{2}{*}{$\%$ inhibition } \\
\hline & Wet weight (mg) & Dry weight (mg) & & \\
\hline DMSO+cotton & $945 \pm 24.50$ & $238 \pm 10.59$ & 707 & - \\
\hline Cotton 50 & $995 \pm 14.35$ & $245 \pm 9.14$ & 750 & - \\
\hline DCL $25+$ cotton & $319 \pm 11.32$ & $118 \pm 7.44$ & 201 & 66 \\
\hline EE $160+$ cotton & $825 \pm 29.37$ & $215 \pm 11.12$ & 610 & 13 \\
\hline EE $320+$ cotton & $604 \pm 10.68$ & $|76 \pm 12.9|$ & 428 & 36 \\
\hline EE $640+$ cotton & $394 \pm 16.99$ & $142 \pm 13.33$ & 252 & 58 \\
\hline
\end{tabular}

Values expressed in milligrams (mean \pm standard deviation). Percentage inhibition of granulomatous tissue formation by ethanol extract and diclofenac was made with respective negative control groups.

\section{Conclusion}

The pragmatic study results concluded that the experiments exhibited novel scientific information because there were no research findings so far as like in our present study. Therefore, this imperative study revealed that the anti-genotoxic and anti-inflammatory activities of ethanol extract of $S$. marginatum was exhibited significantly dose dependent effects however highest activities regarded at the dose of $640 \mathrm{mg} / \mathrm{kgbw}$ than that of other dose tested and their effect was as equal as standard of diclofenac. There are following recent research findings supported and correlated well for the present study that the ethanol extract of $S$. marginatum contained rich phyto nutrients and thereby exhibited highest antibacterial, TAA and RAA over their fractions such as hexane, dichloromethane, ethyl acetate and aqueous fractions. In addition, in-vitro anticancer activity of $S$. marginatum explored through exhibiting $23-80 \%$ cytotoxicity, $11-47 \%$ of apoptosis. Other report stated that $S$. marginatum possessed rich antioxidant, antifungal, and antibacterial as well as cytotoxic activities. ${ }^{2}$

\section{Acknowledgments}

Corresponding author would like to express sincere appreciation to the University Grant Commission (UGC), Government of India, and New Delhi for the contribution and support (TAM -8496) to carry out this research work.

\section{Conflicts of interest}

The authors declare that there are no conflicts of interest.

\section{References}

1. Thirumaran G, Vijayabaskar P, Anantharaman P, et al. Antibacterial and antifungal activities of brown Marine macro alaga (Dictyota dichotoma) from the Gulf of Mannar biosphere reserve. Environ Ecol. 2006;24S:3740 .

2. Arumugam P, Murugan M, Kamalakannan S, et al. Determination of Various Bioactive Potential of Stoechospermum marginatum (C. Agardh) Kutzing in vitro. J Anal Pharm Res. 2017;5(4):145-150.

3. Akbar E, Khakpoor M. Biological activities and chemical composition of solvent extracts of Stoechospermum marginatum (C. Agardh). Acta Bio Pol. 2012;59(4):581-585.

4. Chinnababu B, Purushotham Reddy S, Sankara Rao P, et al. Isolation, semi-synthesis and bio-evaluation of spatane derivatives from the brown algae Stoechospermum marginatum. Bioorg Med Chem Lett. 2015;25(12):2479-2483.

5. Indrawati R, Sukowijoyo H, Limantara L, et al. Encapsulation of brown seaweed pigment by freeze drying characterization and its stability during storage. Procedia chemistry. 2015;14:353-360.

6. Pakyaw S, Wai M, Thida N, et al. The morphology and distribution of Stoechospermum polypodioides (Lamouroux) from Myanmar. Myan Acad Arts Sci. 2009;5(5):239-251. 
7. Anbu A, Arun E, Selvakumari Gopal V. Phytochemical and Pharmacological Profile of Brown Marine Algae Stoecheospermum marginatum. J Acad Indus Res. 2017;5(8):120-122.

8. Flora G, Maria Victorial Rani S. Effect of Stoechospermum marginatum (Ag). Kutz extract on controlling blast disease in rice caused by Pyricularia oryzae under natural field condition. Afr J Agric Res. 2013;8(25):3289-3293.

9. Reka P, Thahira BA, Seethalakshmi M. Nutrient Content, Phytonutrient Composition, Alpha Amylase, Alpha Glucosidase Inhibition Activity and Antioxidant Activity of the Stoechospermum Marginatum Collected in Pre Monsoon Season. Turkish JAF Sci Tech. 2017;5(3):275-280.

10. Arumugam P, Murugan K, Kavipriya R, et al. Antibacterial, antioxidant and anticancer properties of Turbinaria conoides (J. Agardh) Kuetz. Clin Phyto sci. 2017;3:5-10

11. Arumugam P, Ramesh A. Protective effects of solvent fractions of Mentha spicata (L) leaves: evaluated on 4-nitroquinoline-1-oxide induced chromosome damage and apoptosis in mice bone marrow cells. Genet Mol Biol. 2009;32(4):847-852.

12. Arumugam P, Gayatri Priya N, Subathra M, et al. Anti-inflammatory activity of four solvent fractions of ethanol extract of Mentha spicata L. investigated on acute and chronic inflammation induced rats. Environ Toxicol Pharmacol. 2008;26(1):92-95.
13. Kanojia D, Vaidya MM. 4-Nitroquinoline-1-oxide induced experimental oral carcinogenesis. Oral Oncol. 2006;42(7):655-667.

14. Han H, Pan Q, Zhang B, et al. 4-NQO induces apoptosis via p53dependent mitochondrial signaling pathway. Toxicol. 2007;230(23):151-163

15. Diekmann M, Waldmann P, Schnurstein A, et al. On the relevance of genotoxicity for fish populations II: genotoxic effects in zebrafish (Danio rerio) exposed to 4-nitroquinoline-1-oxide in a complete life-cycle test. Aquat Toxicol. 2004;68(1):27-37.

16. Hajhashemi V, Ghannadi A, Hajiloo M. Analgesic and anti-inflammatory effects of Rosa damascena hydroalcoholic extract and its essential oil in animal models. Iran J Pharmaceut Res. 2010;9(2):163-168.

17. Moura ACA, Silva ELF, Fraga MCA, et al. Antiinflammatory and chronic toxicity study of the leaves of Ageratum conyzoides L. in rats. Phytomedicine. 2005;12(1-2):138-142.

18. Gupta V, Kumar P, Bansal P, et al. Anti-inflammatory and Anti-nociceptive Activity of Mitragyna parvifolia. Asian J Med Sci. 2009;1(3):97-99. 